\title{
Graphite, graphene and the flat band superconductivity
}

\author{
G.E. Volovik ${ }^{1)}$ \\ Low Temperature Laboratory, Aalto University, P.O. Box 15100, FI-00076 Aalto, Finland \\ Landau Institute for Theoretical Physics, acad. Semyonov av., 1a, 142432, Chernogolovka, Russia
}

Submitted March 28, 2018

PACS:

Superconductivity with transition temperature $T_{c}=$ 1.7 K has been observed in bilayer graphene 1, 2. The 'main factors, which may shed light on the mechanism of the formation of this superconductivity, are the following. Superconductivity is observed in bilayer graphene, 'when the two layers are twisted, and the maximum of $T_{c}$ takes place at the "magic angle" of twist, at which the electronic band structure becomes nearly flat.

Actually the same factors have been suggested in 'Ref. 3] to explain the experiments in graphite, which reported high-T superconductivity in highly oriented pyrolytic graphite (HOPG) 4, 5, 6, 7, 8. The hints of room-temperature superconductivity are present, only when the sample contains quasi two-dimensional interfaces between the domains of HOPG. 9 These domains should be twisted with respect to each other in order 'to form the flat band in electronic spectrum. 33 This dispersionless energy spectrum has a singular density of 'states, which provides the transition temperature being proportional to the coupling constant instead of the exponential suppression, as was suggested by Khodel and Shaginyan. [10] For nuclear systems the linear dependence of the gap on the coupling constant has been found by Belyaev [11.

There are different potential sources of the formation of the electronic flat band: due to topology [12, 13, 14, 15], due to symmetry [16, 17] and due to interactions [10, 18, 19, 20, 21, 22]. The twist of the graphene layers or HOPG domains provides the topological mechanism of the flat band formation. The situation has the close relation to superconductivity at the interfaces or surfaces of topological crystalline semiconductors, see e.g. 24. The reason of the formation of the nearly flat band can be the spontaneous formation of a misfit dislocation array at the interface. The topological origin of this flat band can be also understood in terms of the pseudo-magnetic field created by strain [25, 26, 27. The topologically protected band touching

\footnotetext{
1) e-mail: volovik(at)ltl.ttk.fi
}

lines in the spectrum of graphite 28, 29, 30 (the socalled Dirac lines) lead to approximate flat band on the surface of graphite or at the interface [12, 13, 14]. The Khodel-Shaginyan mechanism of flattening - the merging of levels due to interaction [10, 18, 19, 20, 21, 22, 23. - is also not excluded, since it leads to the further flattening of the spectrum.

Formation of the superconducting and ferromagnetic states in the flat band materials has been discussed in particular in Refs. [26, 31, 32, 33, 34, 35, 36, 37.

At the moment there are many evidences of enhanced superconducting transition temperature in graphite materials, starting from Refs. [38, 39. The most clear hints of high-T superconductivity in graphite are related to interfaces in HOPG 3, 4, 5, 6, 7, 8. This supports the idea that it is the twisted interface, which is the reason for the flattening of the spectrum. The electron-electron interaction [10, 18, 19] is probably a secondary factor enhancing the flat band singularity in the electronic density of states.

Signatures of high- $T$ superconductivity are also observed in graphite in contact to other materials. Examples are alkanes in contact with a graphite surface [40, 41], polymer composites with embedded graphene flakes 42, 43], films of polystyrene and graphene oxide composite [4], sulfur doped amorphous carbon [45, 46], phosphorus-doped graphite and graphene [47, etc. Signatures of superconductivity with $T_{c}=14 \mathrm{~K}$ have been found on the surface of Grafoil. 48

In conclusion, the observation of superconductivity in graphene supports the specific role played by twist of the graphite planes and by the band flattening. Graphite superconductivity is now becoming the mainstream. One may say that we are coming to graphite era of superconductivity. It is time to combine the theoretical and experimental efforts to reach the bulk room- $\mathrm{T}$ superconductivity in graphite and in similar real or artificial materials. 
I thank T. Heikkilä for fruitful dicussions. This work has been supported by the European Research Council (ERC) under the European Union's Horizon 2020 research and innovation programme (Grant Agreement No. 694248).

1. Yuan Cao, V. Fatemi, Shiang Fang, K. Watanabe, T. Taniguchi, E. Kaxiras and P. Jarillo-Herrero, Unconventional superconductivity in magic-angle graphene superlattices, Nature (2018), doi:10.1038/nature26160.

2. Yuan Cao, V. Fatemi, A. Demir, Shiang Fang, S.L. Tomarken, J.Y. Luo, J.D. Sanchez-Yamagishi, K. Watanabe, T. Taniguchi, E. Kaxiras, R.C. Ashoori and P. Jarillo-Herrero, Correlated insulator behaviour at half-filling in magic-angle graphene superlattices, Nature (2018), doi:10.1038/nature26154.

3. P. Esquinazi, T.T. Heikkila, Yu.V. Lysogorskiy, D.A. Tayurskii, G.E. Volovik, On the superconductivity of graphite interfaces, JETP Lett. 100, 336 (2014).

4. P. Esquinazi, Graphite and its hidden superconductivity, Papers in Physics 5, 050007 (2013).

5. A. Ballestar, J. Barzola-Quiquia, T. Scheike, and P. Esquinazi, Josephson-coupled superconducting regions embedded at the interfaces of highly oriented pyrolytic graphite, New J. Phys. 15, 023024 (2013).

6. A. Ballestar, T.T. Heikkilä, and P. Esquinazi, Interface size dependence of the Josephson critical behaviour in pyrolytic graphite, Supercond Sci. Technol. 27, 115014 (2014).

7. C.E. Precker, P.D. Esquinazi, A. Champi, J. BarzolaQuiquia, M. Zoraghi, S. Muinos-Landin, A. Setzer, W. Böhlmann, D. Spemann, J. Meijer, T. Muenster, O. Baehre, G. Kloess and H. Beth, Identification of a possible superconducting transition above room temperature in natural graphite crystals, New J. Phys. 18, 113041 (2016).

8. M. Stiller, P.D. Esquinazi, J. Barzola-Quiquia and C.E. Precker, Local magnetic measurements of trapped flux through a permanent current path in graphite, J. Low Temp. Phys. 191, 105-121 (2018).

9. P.D. Esquinazi, Reply to the Commentary on "Graphite and its hidden superconductivity", Papers in Physics 5, 050009 (2013).

10. V.A. Khodel and V.R. Shaginyan, Superfluidity in system with fermion condensate, JETP Lett. 51, 553 (1990).

11. S.T. Belyaev, On the nature of the first excited states of even-even spherical nuclei, JETP 12, 968-976 (1961).

12. T.T. Heikkilä and G.E. Volovik, Flat bands as a route to high-temperature superconductivity in graphite, in: $B a$ sic Physics of Functionalized Graphite, Springer 2016, pp. $123-143$.

13. T.T. Heikkilä, N.B. Kopnin and G.E. Volovik, Flat bands in topological media, JETP Lett. 94, 233-239 (2011).
14. T. Hyart, R. Ojajärvi and T.T. Heikkilä, Two topologically distinct drac-line semimetal phases and topological phase transitions in rhombohedrally stacked honeycomb lattices, J. Low Temp. Phys. 191, 35-48 (2018).

15. H.K. Pal, S. Spitz and M. Kindermann, Emergent geometric frustration and flat band in moire bilayer graphene, arXiv:1803.07060

16. E.H. Lieb, Two theorems on the Hubbard model, Phys. Rev. Lett. 62, 1201-1204 (1989).

17. R. Bistritzer and A.H. MacDonald, Moire bands in twisted double-layer graphene, PNAS 108, 12233-12237 (2011).

18. G.E. Volovik, A new class of normal Fermi liquids, JETP Lett. 53, 222-225 (1991).

19. P. Nozieres, Properties of Fermi liquids with a finite range interaction, J. Phys. (Fr.) 2, 443 (1992).

20. D. Yudin, D. Hirschmeier, H. Hafermann, O. Eriksson, A.I. Lichtenstein, M.I. Katsnelson, Fermi condensation near van Hove singularities within the Hubbard model on the triangular lattice, Phys. Rev. Lett. 112, 070403 (2014).

21. G.E. Volovik, On Fermi condensate: near the saddle point and within the vortex core, JETP Lett. 59, 830 (1994).

22. A.A. Shashkin, V.T. Dolgopolov, J.W. Clark, V.R. Shaginyan, M.V. Zverev and V.A. Khodel, Merging of Landau levels in a strongly-interacting two-dimensional electron system in silicon, Phys. Rev. Lett. 112, 186402 (2014).

23. M.Yu. Melnikov, A.A. Shashkin, V.T. Dolgopolov, S.-H. Huang, C.W. Liu, S.V. Kravchenko, Indication of band flattening at the Fermi level in a strongly correlated electron system, Scientific Reports 7, 14539 (2017).

24. E. Tang and L. Fu, Strain-induced partially flat band, helical snake states, and interface superconductivity in topological crystalline insulators, Nature Phys. 10, 964 (2014).

25. F. de Juan, J. L. Manes, and M.A.H. Vozmediano, Gauge fields from strain in graphene, Phys. Rev. B 87, 165131 (2013).

26. V.J. Kauppila, F. Aikebaier, and T.T. Heikkilä, Flatband superconductivity in strained Dirac materials, Phys. Rev. B 93, 214505 (2016).

27. A. Ramires, J. L. Lado, Electrically tunable gauge fields in tiny-angle twisted bilayer graphene, arXiv:1803.04400

28. G.P. Mikitik and Yu.V. Sharlai, Dirac points of electron energy spectrum, band-contact lines, and electron topological transitions of $3 \frac{1}{2}$ kind in three-dimensional metals, Phys. Rev. B 90, 155122 (2014).

29. G.P. Mikitik and Yu.V. Sharlai, Band-contact lines in the electron energy spectrum of graphite, Phys. Rev. B 73, 235112 (2006).

30. G.P. Mikitik and Yu.V. Sharlai, The Berry phase in graphene and graphite multilayers, Low Temp. Phys. 34, 794-780 (2008). 
31. N.B. Kopnin, Surface superconductivity in multilayered rhombohedral graphene: Supercurrent, JETP Lett. 94, 81 (2011).

32. N.B. Kopnin, M. Ijäs, A. Harju, T.T. Heikkilä, Hightemperature surface superconductivity in rhombohedral graphite, Phys. Rev. B 87, 140503(R) (2013).

33. Long Liang, T.I. Vanhala, S. Peotta, T. Siro, A. Harju and P. Torma, Band geometry, Berry curvature, and superfluid weight, Phys. Rev. B 95, 024515 (2017).

34. R. Ojajärvi, T. Hyart, M. Silaev, T.T. Heikkilä, Competition of electron-phonon mediated superconductivity and Stoner magnetism on a flat band, arXiv:1801.01794

35. H. Tasaki, Ferromagnetism in the Hubbard models with degenerate single-electron ground states, Phys. Rev. Lett. 69, 1608 (1992).

36. A. Mielke and H. Tasaki, Ferromagnetism in the Hubbard model, Commun. Math. Phys. 158, 341-371 (1993).

37. T. Löthman and A.M. Black-Schaffer, Universal phase diagrams with superconducting domes for electronic flat bands, Phys. Rev. B 96, 064505 (2017).

38. Y. Kopelevich, V.V. Lemanov, S. Moehlecke, and J.H.S. Torres, Landau level quantization and possible superconducting instabilities in highly oriented pyrolitic graphite, Phys. Solid State 41, 1959-1962 (1999).

39. Y. Kopelevich, P. Esquinazi, J. H. S. Torres, and S. Moehlecke Ferromagnetic- and superconducting-like behavior of graphite, J. Low Temp. Phys. 119, 691-702 (2000).

40. Y. Kawashima, Possible room temperature superconductivity in conductors obtained by bringing alkanes into contact with a graphite surface, AIP Advances 3, 052132 (2013).

41. Y. Kawashima, Observation of the Meissner effect at room temperature in single-layer graphene brought into contact with alkanes, arXiv:1801.09376

42. A.N. Ionov, Josephson current-voltage characteristic of a composite based on polystyrene and graphene oxide Pis'ma ZhTF 41, 79-85 (2015); Techn. Phys. Lett. 41, 651 (2015).

43. A.N. Ionov, Josephson-like behavior of the currentvoltage characteristics of multi-graphene flakes embedded in polysterene, J. Low Temp. Phys. 185, 515 (2016).

44. A.R. Khairullin, M.N. Nikolaeva, A.N. Bugrov, Resistance of composite films based on polystyrene and graphene oxide, Nanosystems 7, 1055-1058 (2016).

45. R.R. da Silva, J.H.S. Torres, and Y. Kopelevich, Indication of superconductivity at $35 \mathrm{~K}$ in graphite-sulfur composites, Phys. Rev. Lett. 87, 147001 (2001).

46. I. Felner, Peculiar magnetic features and superconductivity in sulfur doped amorphous carbon, Magnetochemistry 2, 34 (2016).

47. G. Larkins, Y. Vlasov and K. Holland, Evidence of superconductivity in doped graphite and graphene, Supercond. Sci. Technol. 29, 015015 (2016).
48. Frank Arnold PhD (Royal Holloway University of London), 2015, and private communication J. Saunders. 